\title{
THE UNITED LAUNCH ALLIANCE DELTA IV VEHICLE - AN UPDATE TO THE PULSE SETTLING PROPELLANT MANAGEMENT APPROACH
}

\section{D. Berglund, Ch. E. Bassett, J. Mishic, and D. Schrage}

\begin{abstract}
During the flight readiness review process of a recent Delta IV mission, an independent review team from Analex Corporation presented results of simulations of the behavior of the second-stage cryogenic propellants. These results differed considerably from those Boeing and the Aerospace Corporation were showing at the time. A team consisting of representatives from Boeing, the Air Force, the National Reconnaissance Office Office of Space Launch (NRO/OSL), the Aerospace Corporation, Flow Sciences, and Analex investigated this slosh behavior and was chartered to resolve the discrepancy between analysis results. During the investigation, it was concluded that the slosh behavior of liquid hydrogen $\left(\mathrm{LH}_{2}\right)$, during the second stage coast phase of flight, would not be constrained to the aft end of the tank and thus presented an increased mission risk. The presence of propellant in the tank forward dome at tank venting could lead to propellant ingestion into the vent/relief system and result in a thrust imbalance. This paper is an update to a paper previously released [1] that focused on the team's investigative efforts and resolution of the issues associated with the sloshing, including the implementation of a pulse-settling approach for propellant management. The update (italicized text) includes a discussion of the flight validation of the successful launches that implemented pulse-settling. Figure 1 is a screen-shot from a simulation of the $\mathrm{LH}_{2}$ sloshing in the second-stage tank during a coast period.
\end{abstract}

\section{INTRODUCTION}

The Delta family of launch vehicles (LV) (Fig. 2) has continued to evolve throughout its 40-year legacy to meet customers' growing needs. The successful Delta IV development represents the most dramatic change in capability during this Delta legacy.

This is an Open Access article distributed under the terms of the Creative Commons Attribution-Noncommercial License 3.0, which permits unrestricted use, distribution, and reproduction in any noncommercial medium, provided the original work is properly cited. 


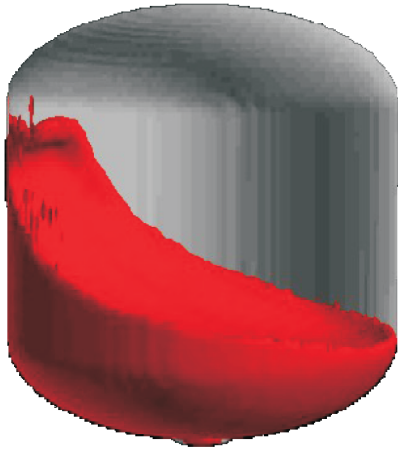

Figure 1 Simulated $\mathrm{LH}_{2}$ slosh. (Refer Berglund et al., p. 294.)
As shown in Fig. 2, Delta IV adds five vehicles to the Delta family: the Delta IV Medium, three Delta IV Medium vehicles with solid strapon rocket motors (Medium-Plus variants), and the Delta IV Heavy with two strap-on common booster cores (CBC) serving as liquid rocket boosters.

The Delta IV family is built on a solid foundation of heritage hardware and proven processes in manufacturing, quality, engineering, and supplier management. The Delta IV family evolves to expand the Delta capability while at the same time creating a robust system with improvements in producibility and operability. The primary avionics system, the 4-meter fairing, the 4-meter cryogenic second-stage tanks,

Delta II
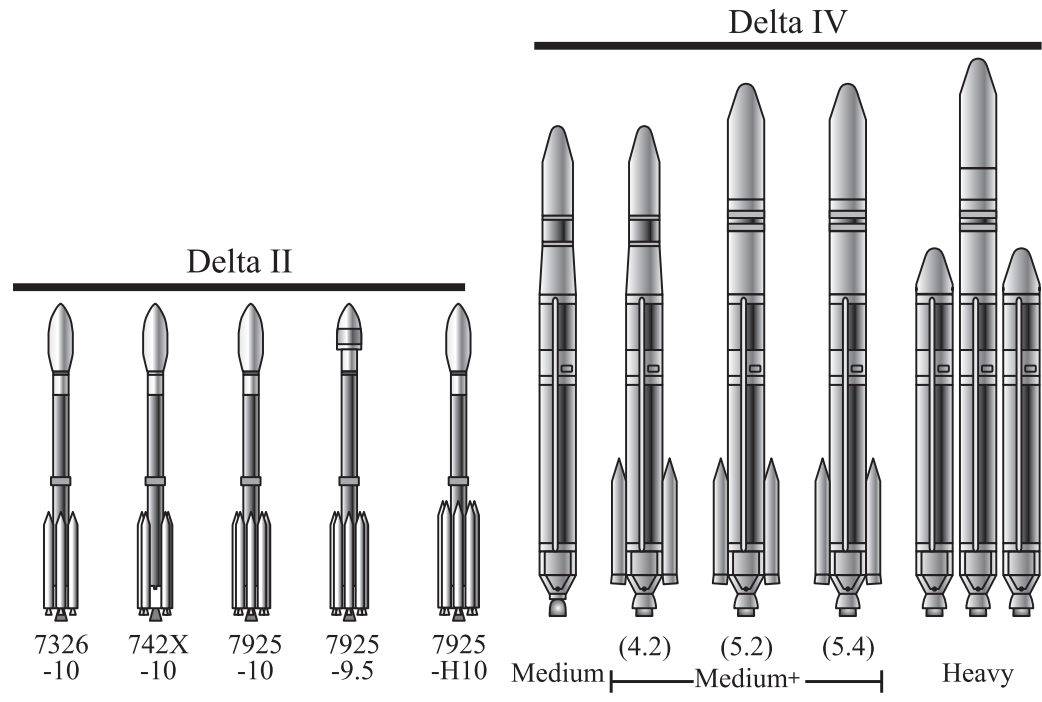

\begin{tabular}{|lrrrrrrrrrr|}
\hline GTO, $\mathrm{kg}$ & 890 & 1,030 & 1,760 & 1,830 & 2,130 & 4,230 & 5,940 & 4,870 & 6,820 & 12,760 \\
\hline LEO $\mathrm{kg}$ & 2,470 & 2,880 & 4,600 & 4,780 & 5,650 & 9,110 & 12,300 & 10,620 & 13,870 & 21,890 \\
\hline GEO, $\mathrm{kg}$ & & & & & & 1,140 & 2,040 & 1,690 & 2,790 & 6,280 \\
\hline
\end{tabular}

GTO $=185 \times 35,786 \mathrm{~km}$ at $28.7^{\circ}$ Delta II and $27.0^{\circ}$ Delta IV.

$\mathrm{LEO}=407 \mathrm{~km}$ circular at $28.7^{\circ}$.

$\mathrm{GEO}=35,786 \mathrm{~km}$ circular at $0^{\circ}$

Figure 2 Family of Delta launch vehicles (GTO - geosynchronous transfer orbit; LEO - low Earth orbit; and GEO - geostationary orbit) 
and the second-stage engine are examples of heritage hardware carried into the Delta IV design. In addition, the strap-on solid rocket motors are derived from the smaller diameter solids used on Delta II and Delta III.

All configurations of the Delta IV family share the same first stage, the CBC. The CBC consists of the interstage, liquid oxygen $\left(\mathrm{LO}_{2}\right)$ tank, centerbody, $\mathrm{LH}_{2}$ tank, engine section, and the U.S.-developed Pratt \& Whitney Rocketdyne RS-68 engine.

The RS-68 engine, clean and environmentally friendly, utilizes $\mathrm{LO}_{2}$ and $\mathrm{LH}_{2}$ propellants producing more than $2,918 \mathrm{kN}$ of thrust (sea level).

The Medium-Plus variants consist of a CBC and either two or four 1.5meter-diameter graphite-epoxy solid propellant strap-on motors. These motors are designed and manufactured by Alliant Techsystems and have both fixed and vectorable nozzle configurations. The Medium-Plus variants include either a 4 or 5-meter-diameter fairing.

The second-stage Pratt \& Whitney Rocketdyne RL10B-2 engine derives its power from $\mathrm{LO}_{2}$ and $\mathrm{LH}_{2}$ cryogenic propellants as well and is used on all Delta IV configurations. Producing $110 \mathrm{kN}$ of thrust, the engine possesses an extendible nozzle designed for boost-phase environments and longer second-stage burn durations.

\section{Flight Readiness Review Process}

Before each launch, the Delta IV vehicles are subjected to a rigorous review process to ensure mission success. The Delta IV flight readiness review process was derived from the flight-proven Delta II and Titan IV payload faring launch readiness review process.

This disciplined process integrates functions from quality assurance, manufacturing, launch site processing, and engineering. The process consists of a series of reviews scheduled to validate and establish with a high degree of confidence that the LV and ground support equipment will perform reliably and meet mission objectives.

Reviews are linked to key Delta IV launch milestones (Fig. 3). The process progresses from production through postlaunch data reviews and includes customers and independent assessment teams.

During the flight readiness review process of a recent Delta IV mission, an independent review team from Analex Corporation presented results of simulations of the behavior of the second-stage cryogenic propellants. The Analex Corporation was performing this independent analysis as part of a contract with the NRO/OSL for EELV missions. These results differed considerably from those of Boeing and the Aerospace Corporation. Boeing then recommended that the launch team delay further vehicle processing until the discrepancy between analysis results was resolved. 


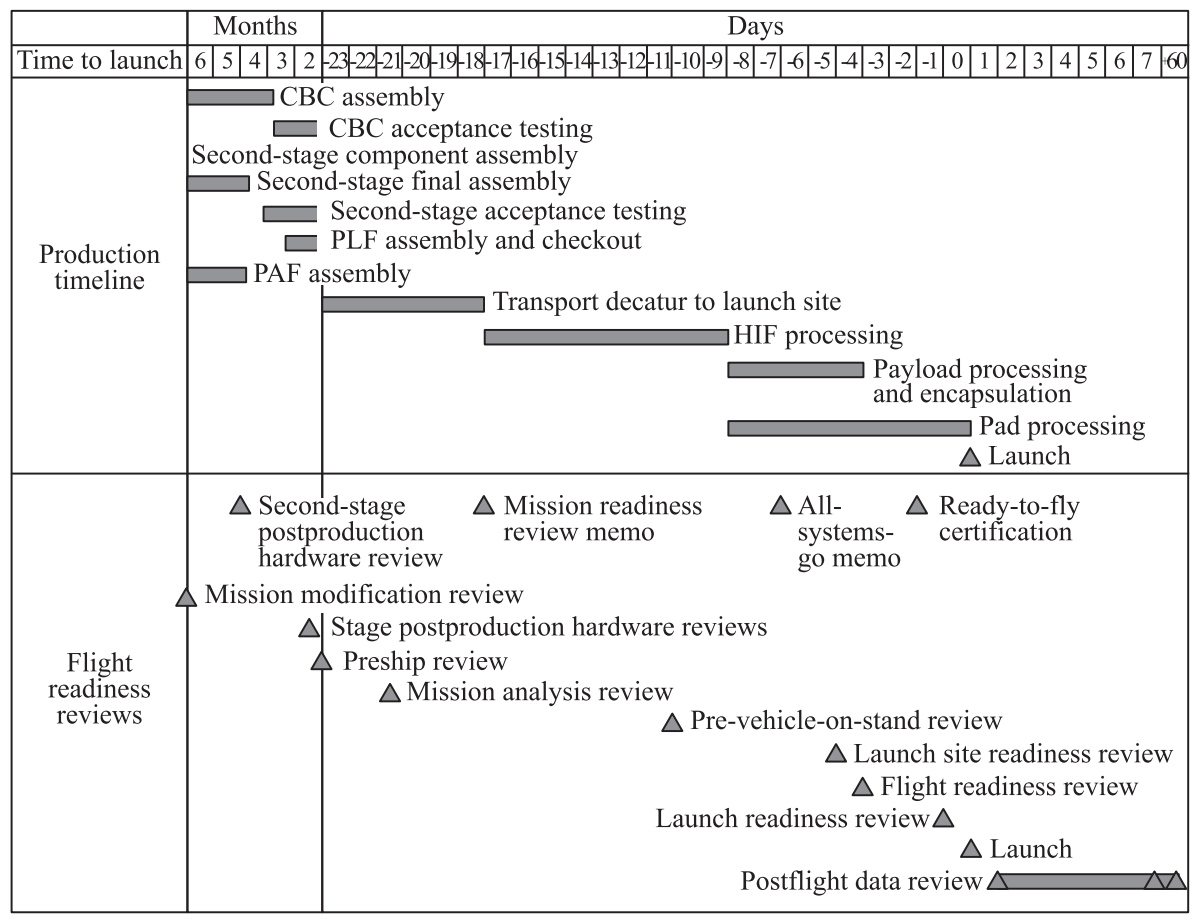

Figure 3 Delta IV flight readiness schedule (generic) (PLF — payload fairing and PAF - payload attach fitting)

\section{PROBLEM}

The Boeing Company, early in the LV and satellite integration cycle, analyzed the behavior of the second-stage cryogenic propellants, including sloshing behavior. The original Boeing analysis indicated acceptable sloshing of propellants within the tanks. However, an Analex independent analysis indicated excessive $\mathrm{LH}_{2}$ sloshing after second-stage engine cutoff (SECO) and during the coast turn to a flight attitude known as the passive thermal control (PTC).

To perform this analysis, both Boeing and Analex used the engineering computational fluid dynamics (CFD) tool FLOW-3D. However, the Boeing analysis used only a four-degree-of-freedom (DOF) tank dynamic model, whereas Analex used a full six-DOF model, which included the lateral forces created by the second-stage attitude control system (ACS).

Under worst-case assumptions, Analex analysis indicated that $\mathrm{LH}_{2}$, during the second stage turn to PTC, would not be constrained in the aft end of the tank. Because of the risk of unplanned second-stage $\mathrm{LH}_{2}$ venting during this phase of 
flight, the lack of propellant control could lead to propellant ingestion into the tank vent-and-relief system. This process could result in a thrust imbalance and loss of vehicle control.

\section{INVESTIGATION}

Immediately following the decision to halt vehicle processing, a multiagency team was created to reconcile analysis differences and determine corrective action for subsequent Delta IV missions.

The team consisted of representatives from Boeing, the Air Force, the NRO/ OSL for EELV missions, the Aerospace Corporation, Flow Sciences, and Analex. The charter of the team was to "reconcile differences between Boeing and Analex simulation models via fault tree analysis" and "determine proper methodology for future Delta IV second-stage propellant slosh analysis." For the team to be successful, all fault tree items would need to be dispositioned and the updated slosh predictions match at a level of flow detail consistent with mission assurance.

To define the methodology to be used in future mission slosh analyses, team consensus would be needed, establishing a baseline simulation modeling method and a suite of off-nominal dispersion cases (credible worst-case scenarios). The goal of the team was to maintain acceptable propellant control in the aft end of the tank to help ensure mission success.

\section{Results}

The team presented conclusions and status of its progress through a series of engineering review boards (ERB). The team, during the investigation, concurred that the independent analysis results did show potential flight scenarios with an unacceptable level of risk to mission success.

After creation of a fault tree, evaluation of the branches was conducted to determine the likelihood of causing the noted differences. The credible causes of the different analysis outcomes were all linked to differences in dynamic inputs into each of the models. At the conclusion of the fault tree investigation, the team was able to reconcile the differences by running identical dynamic input files. Thus, it was determined that the root cause of the different outcomes was due solely to the dynamic assumptions. The initial Boeing result, the initial Analex result, and the Boeing confirmation of the dynamic input as root cause of the difference in analysis outcome is shown in Fig. 4.

The team turned its focus to establishing the right flight simulations to run, which would bound credible worst-case scenarios and help redesign the flight sequence of events. A matrix of flight conditions was formed to establish the cases 

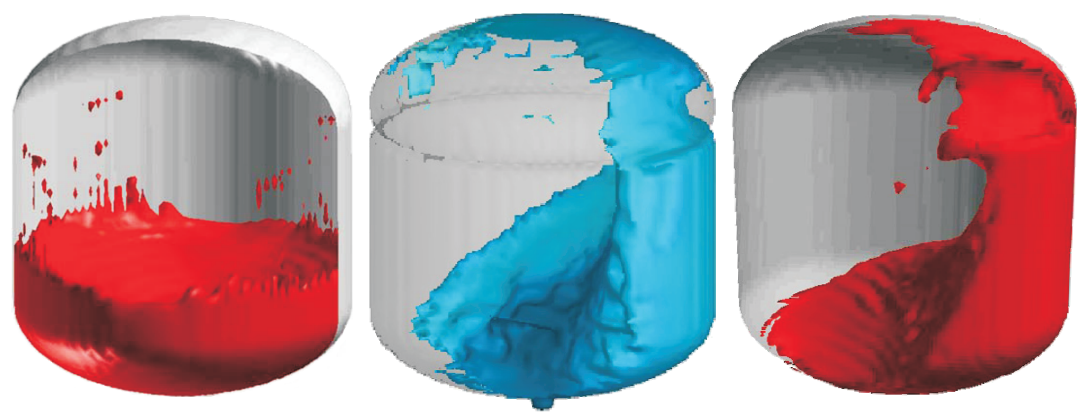

Figure 4 Original Boeing $\mathrm{LH}_{2}$ slosh analysis results, Analex independent results, and Boeing confirmation of input dynamics as the source of analysis disagreement (left to right). (Refer Berglund et al., p. 298.)

to be simulated, including worst-case second-stage engine ignition dynamics, dispersed propellant masses, and worst-case phasing of propellant dynamics.

During these simulations, several observations were noted.

A key facet of the selected propellant management method was the implementation of a pulse-settling process in controlling slosh amplitude.

\section{PULSE-SETTLING APPROACH TO PROPELLANT MANAGEMENT}

Second-stage vehicle and propellant control during coast phases is managed by the ACS. The ACS consists of four modules, each positioned at $90^{\circ}$ from the other, near the aft end of the stage (Fig. 5). Each module contains three hydrazine-powered thrusters, two pointed in the plane normal to the vehicle axis, and a third pointing aft along the vehicle axis. The former pair is used for vehicle control (pitch, yaw, and roll) and the latter is used for propellant-settling and for stage translation maneuvers during coast. To maintain balanced thrust, the axial thrusters must be used in pairs, either two thrusters in opposite-positioned modules or all four at once.

The aft thrusters are normally sequenced at 4-ON following SECO, reduced to 2-ON later, and finally all-OFF even later. The final transition to all-OFF leaves a small axial thrust on the stage from the continuous vent system (CVS). It was found that during these transitions, slosh energy was amplified (slosh amplitude increased), as would be expected from the analogy of a pendulum transitioning to reduced gravity levels. In a simple pendulum case, the optimum transition point to avoid an increase in slosh amplitude would be the point of highest amplitude (maximum potential energy, minimum kinetic energy). However, given the 


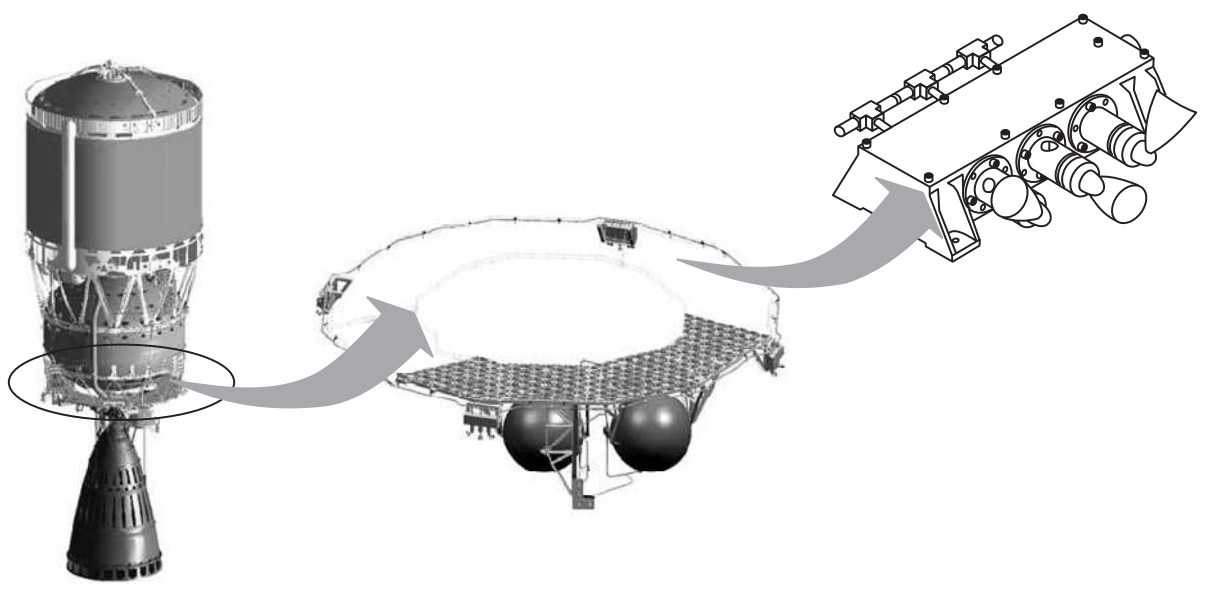

Figure 5 Attitude control system

uncertainty in various events that influence the slosh period relative to mission time, it was apparent that attempting to time the transitions was not practical.

To reduce the probability of occurrence of a large slosh amplification during a single settling thrust transition, several intermediate thrust levels were needed. The only practical way to achieve such a variation (with fixed hardware configuration) was to pulse the thrusters at various $\mathrm{ON}-\mathrm{OFF}$ periods. For example, to achieve 804-ON thrust to CVS thrust.

The benefit from these increased transitions was two-fold. First, the probability of making a transition at the worst slosh condition (maximum kinetic energy) during a slosh period was reduced substantially. For example, to equal the effect of a single worst-case reduction from 4-ON thrust to 2-ON thrust, all four intermediate transitions would have to happen at worst-case times. The second benefit arose from the scenario of reducing thrust in increments, for example, from $4-\mathrm{ON}$ to $2-\mathrm{ON}$, by increasing the time during which slosh damping occurs. Figure 6 shows the slosh analysis result using a set of worst-case assumptions before and after implementing a pulse-settling sequence.

An important part of accepting the pulse-settling approach was the review process by the second-stage team functional constituents: controls, avionics, thermal, guidance, and propulsion. It was determined that thruster qualification limits would not be exceeded by the planned operations and that vehicle consumables would not be adversely affected. (In fact, it is noteworthy that the pulse-settling plan can be implemented with no increase in ACS hydrazine usage over the previous settling plan.) Further, implementing the sequence of thrust pulses would be neither a costly nor impractical use of the vehicle computer systems. 


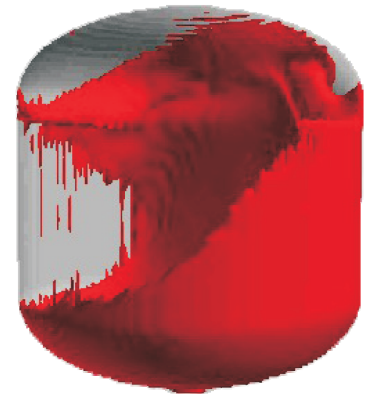

(a)

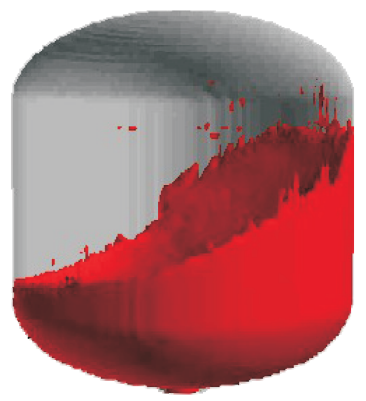

(b)

Figure 6 Worst-case $\mathrm{LH}_{2}$ slosh before $(a)$ and after $(b)$ implementing a pulse-settling sequence. (Refer Berglund et al., p. 300.)

The following section provides additional details on the computational propellant slosh modeling of Analex and the development of next-generation CFD tooling to successfully simulate the entire LV mission.

\section{ANALEX COMPUTATIONAL PROPELLANT SLOSH MODELING}

Slosh refers to the bulk motions of fuel and oxidizer inside a propellant tank. Possible deleterious effects of excessive slosh can include liquid ingestion at vent ports, cavitation in the main engine pump, and ullage pressure collapse. In the past, preflight prediction of tank slosh was derived from terrestrial testing, which did not replicate flight conditions such as the wide range of LV acceleration environments. Early CFD work successfully simulated parts of the LV flight sequence, but the development of next-generation CFD tooling to successfully simulate the entire LV mission is a recent event.

Analex CFD slosh modeling is an example of this next-generation, Sim-LikeYou-Fly (SLYF) technique (Fig. 7). Model development begins with importing detailed tank geometry (Fig. 8) in bulk data format (BDF) generated by NASTRAN or various other commercial tools. The tanks are rendered in a highly detailed fashion and include internal features such as antivortex baffles, antigeyser plates, slosh baffles, and exit ports.

Fluid properties such as density, viscosity, and surface tension are then applied to the fluid as in other CFD schemes. The model becomes trans-disciplinary as trajectory inputs are added. These include propellant initial fill level and extraction flow as well as 6-DOF inputs: flight-like tank acceleration and tank 


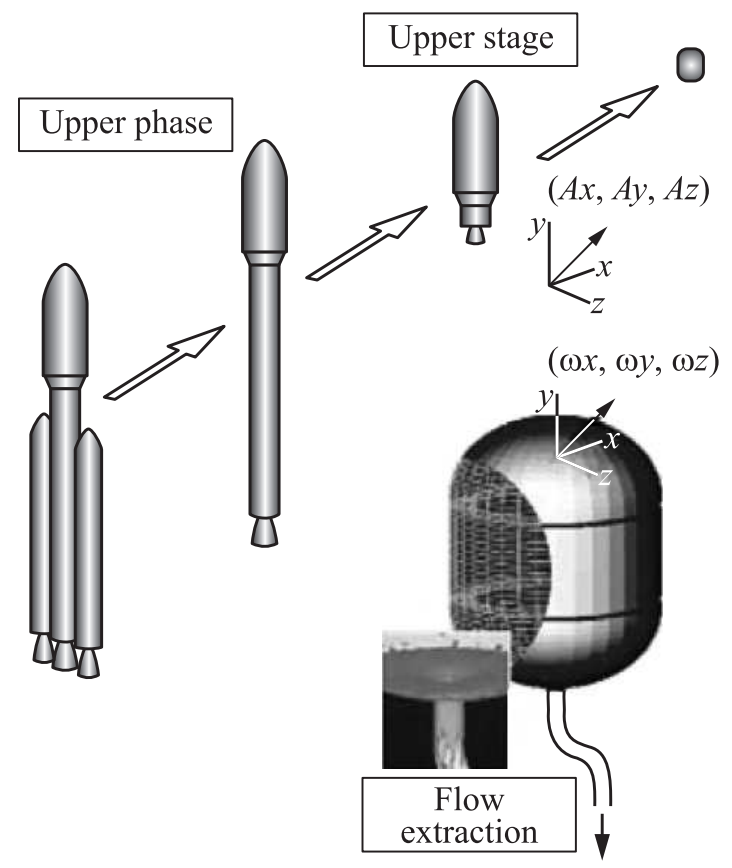

Figure 7 Sim-Like-You-Fly technique

rotation. Analex employs a number of in-house routines to visualize tank motions as a model check. The important difference in this modeling technique is that the tank is simulated from liftoff through end-ofmission; the simulation does not "jump in" at an arbitrary point. This is critical because prehistory events play a large part in determining "downstream" slosh features.

The analysis treats propellant tanks in both LV stages. Of note, the considerations for both stages vary greatly. In the booster, accelerations vary from $1 g$ to $5 g$ and the primary

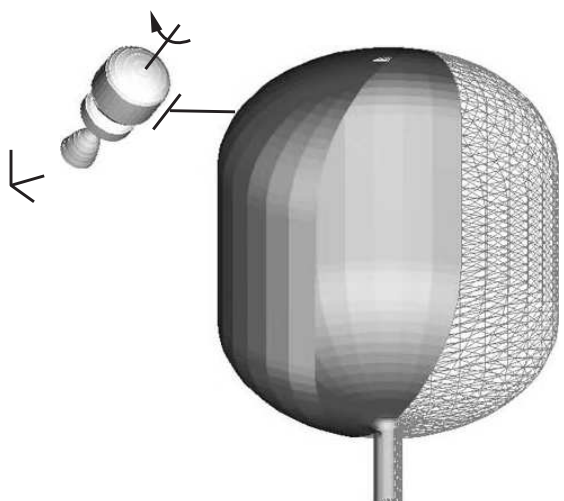

Figure 8 Sample tank geometry concern is premature vapor ingestion at the engine inlet prior to engine cutoff. In the upper stage, acceleration can be in the milli-g range. In addition to vapor 

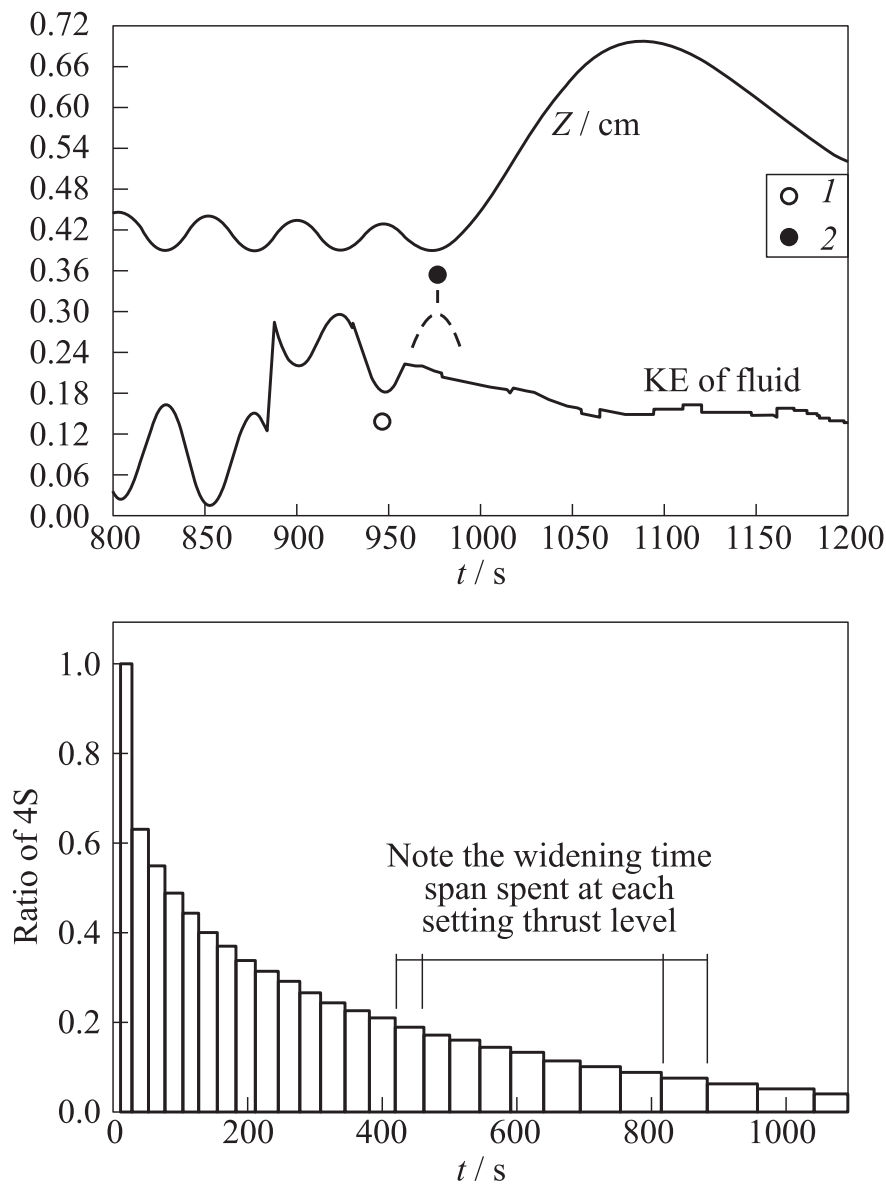

Figure 9 Comparison of MIN (1) and MAX (2) KE breakpoints and QS design with expanding time at each thrustbreak

ingestion, improper thermodynamic conditioning and/or the presence of liquid at the vent ports can occur. The Analex SLYF technique predicts the slosh environment over this entire regime. Typical analysis outputs include animation of the fluid free surface (interface between liquid and vapor phase), slosh and pull-through height, fluid kinetic energy (KE), and reaction loads.

In addition to predicting slosh magnitude, the SLYF technique can be used to develop a plan for slosh mitigation. An example of this is the QS $(\mathrm{Q}=$ any integer; $\mathrm{S}=$ seconds) breakpoint settling scheme developed as a part of the slosh mitigation process (Fig. 9) of a recent Delta IV mission. Prior to this mission, it was found that upper-stage slosh during coast periods would be magnified if 


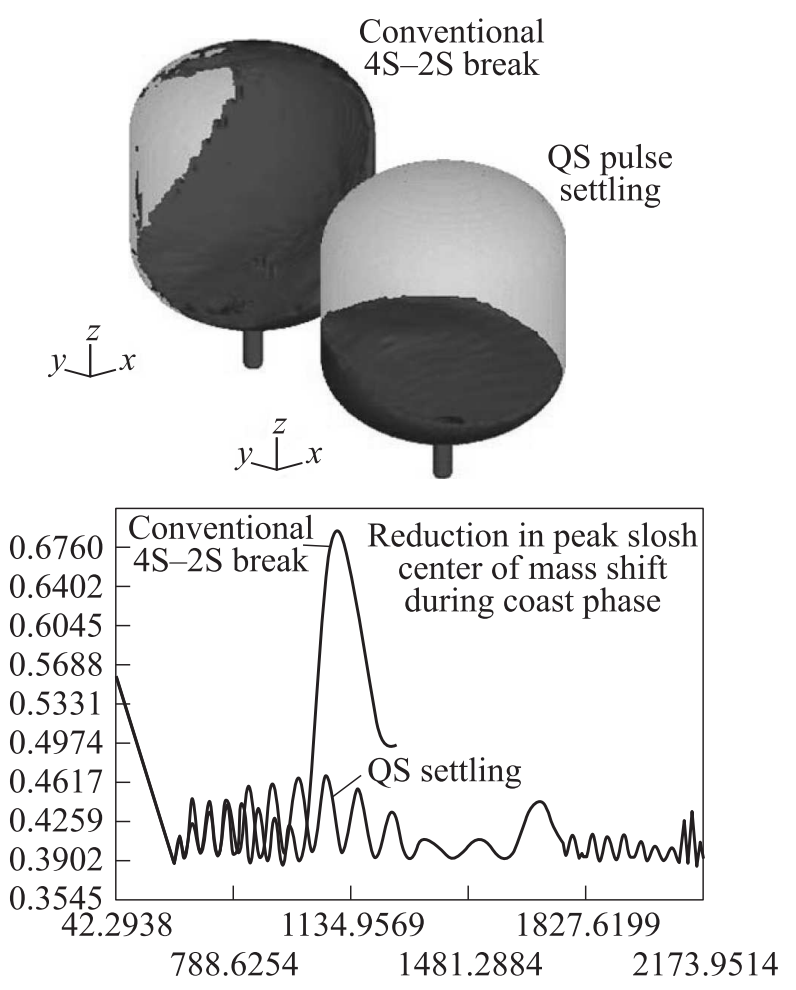

Figure 10 Comparison of fluid free surface for standard and QS setting

vehicle deceleration breakpoints coincided with local maxima in the mean fluid KE. The QS scheme essentially decelerated the LV in a series of small steps, greatly reducing the chance of lining up all decelerations with maximum KE points. The spacing of the steps depends on the slosh frequency, which can also be determined by CFD. Figure 10 shows a conventional $4 \mathrm{~S}-2 \mathrm{~S}$ break scheme. The plot shows the KE of the fluid in motion. Proper settling design should set the location of the thrust break ( $4 \mathrm{~S}$ to $2 \mathrm{~S}$ in this instance) at the MIN KE breakpoint. However, this point is not known a priori.

The QS methodology treats the problem in a statistical manner by creating multiple thrust breakpoints and by setting the location of the breakpoints to statistically span an average of MIN and MAX KE loci. This relieves the system of large slosh. As a result, a proposed settling sequence for Delta was developed that greatly reduced slosh magnitude. The actual settling sequence used for a recent Delta IV mission was different than the original one described by Analex due to other resource concerns, primarily hydrazine usage. However, a settling routine following the basic QS settling principles was employed. 


\section{FLIGHT VALIDATION}

Three missions have flown utilizing the updated analysis process that came from the slosh investigation. Although only one of these flights possessed instrumentation (wall sensors) that was specific to the evaluation of slosh behavior in coast phases, all three missions were free of slosh-related issues. The single instrumented mission (GOES-N) supplied data that confirmed slosh behavior in the $\mathrm{LH}_{2}$ tank during long coast was essentially bounded by the prelaunch forecast.

\section{CONCLUDING REMARKS}

A diverse government/industry team functioned well to resolve a flight-critical concern and return the Delta IV to flight readiness. The subsequent work product of the team resulted in new observations not previously known to the participants and in development of a process that increases the probability of mission success.

The source of the lack of second-stage coast slosh analysis agreement between Boeing and Analex was determined to be due to a difference in the input dynamics driving second-stage coast slosh behavior.

Results of the subsequent analysis investigation showed that several factors contribute to propellant behavior during coast periods, but the essential candidate to improve slosh behavior is the implementation of intermediate levels of settling thrust. This implementation has been successfully realized by utilizing pulse-settling, which reduces the probability of a badly timed thrust transition and increases slosh damping time.

\section{REFERENCES}

1. Berglung, M. D., Ch. E. Bassett, J. K. Kelso, J. Mishic, and D. Schrage. 2007. The Boeing Delta IV launch vehicle - pulse-settling approach for second-stage hydrogen propellant management. Acta Astronautica 61(1-6):416-24. 